\title{
Identification of Ploidy Variation of Ponkan Embryo Rescue Plants
}

Luo Sainan, Zhang Wen, Chen Peng, Han Jian, Li Feifei, Li Xianxin $\square$

Hunan Academic of Agricultural Sciences, Horticultural Institutute, Changsha, 410125

D Corresponding author Email: nkylxx118@163.com

Molecular Plant Breeding, 2020, Vol.11, No.28 doi: $10.5376 / \mathrm{mpb} .2020 .11 .0028$

Received: 27 Jul., 2020

Accepted: 25 Nov., 2020

Published: 31 Dec., 2020

Copyright $\odot 2020$ Luo et al., This article was first published in Molecular Plant Breeding in Chinese, and here was authorized to translate and publish the paper in English under the terms of Creative Commons Attribution License, which permits unrestricted use, distribution, and reproduction in any medium, provided the original work is properly cited.

Preferred citation for this article:

Luo S.N., Zhang W., Chen P., Han J., Li F.F., and Li X.X., 2020, Identification of ploidy variation of ponkan embryo rescue plants, Molecular Plant Breeding, 11(28): 1-8 (doi: $\underline{10.5376 / \mathrm{mpb} .2020 .11 .0028)}$

\begin{abstract}
China has abundant of local citrus resources, which had many seeds. The Seedless is the aim of the local citrus breeding. Triploid citurs resources usually obtained by crosssing breeding between the diploid and tetraploid plants. However, the resources of tetraploid citrus are limited. It has been reported that the hybrid between diploid and diploid plants can produce triploid citrus, which not only saves the breeding time, but also shortens the breeding cycles. In this project, flow cytometry detection assisted by embryo rescue system and SSR identification technology were used to improve the probability of obtaining polyploidy from the hybrid offspring, and provide technical support for obtaining polyploidy from large-scale hybridization between diploid and diploid. plants. Three diploid pollen cultivars were crossbred with Hunan local cultivar 8306, immature seeds were collected for embryo rescue to obtain regenerative plants, plant ploidy was detected by flow cytometry and stoma electron microscope, and offspring genotypes were identified by SSR. After 100 d hybrid between three diploid pollens and Ponkan 8 306, 405 plants were obtained by embryo rescue technique. And 70 plants survived in the greenhouse after transplantation. Through flow cytometry instrument and stomatal electron microscope inspection, the results showed that SSR analysis validated that there are 13 polyploid plants, including 1 tetraploid plant and 12 triploid plants. The ratio of polyploidy was $18.57 \%$. This study is obtained triploid and tetraploid plants by Ponkan embryo rescue from diploid interspecific hybridization. In this study, the embryo rescue technology system and the early identification system for polyploid hybridization were established, and a lot of new polyploid germplasm were obtained.
\end{abstract}

Keywords Embryo rescue; Flow cytometry; SSR; Ponkan; Polyploid

Ponkan (Citrus. reticulata Blanco cv. Ponkan) is one of the three pillar industries of Citrus industry in Hunan Province. Its fruit is sweet and sour with strong mastication and good storability, which has been favored by consumers (Deng, 2005; Zhou and Ye, 2010). However, Ponkan has many seeds. So, breeding new varieties with seedless is the primary goal of Ponkan breeding. Because triploid is highly sterile and produces seedless fruit, it is an important way to cultivate triploid to obtain seedless citrus (Deng, 1996). Conventional seedless type selection is mainly carried out through bud mutation, seed selection and radiation mutagenesis breeding. Due to the rise of biotechnology and the use of conventional sexual hybridization assisted biotechnology, triploid breeding has achieved unprecedented development (Yi and Deng, 1998). There have been many reports on the large-scale creation of citrus triploid by conventional sexual hybridization with diploid female parent and tetraploid male parent (Xie et al., 2014). However, some previous studies have shown that some diploid citrus varieties can produce small seeds after interspecific hybridization or natural pollination, the size of which is $1 / 6 \sim 1 / 3$ of normal seeds, and most of them are triploid (Chen and Hu, 1981). Wang and $\mathrm{Li}$ (2007) reported that triploid could be obtained from undeveloped ovules of mature local early fruits after in vitro embryo culture, with the frequency of $3.12 \%$. Therefore, exploring the triploid produced by diploid interspecific hybridization is one of the shortcuts to cultivate triploid plants, which not only saves the time of breeding tetraploid parents, but also overcomes the problem that tetraploid meiosis tends to produce aneuploid (Guo et al., 1997).

In this study, with ponkan as the female parent, we hope to use diploid and diploid hybrid breeding technology, assisted embryo rescue technology to improve the survival rate of immature embryos or abortive embryos, and use flow cytometry and stomatal scanning electron microscopy to identify the early ploidy of seedlings, so as to 
quickly obtain polyploid progenies, and use assisted SSR molecular markers to identify the homozygous genotype and heterozygous genotype. The embryonic rescue technique system and the early identification system of polyploid obtained by interspecific hybridization of polyploid citrus were established in this study, which could provide theoretical basis for the breeding of new seedless varieties and the basic research of hybridization.

\section{Results and Analysis}

\subsection{Pollination, embryo rescue and plant regeneration}

Three diploid interspecific hybridization combinations were designed to rescue the regenerated plants (Figure 1) by pollination and embryo rescue techniques. In combination 1,8 fruits were obtained with a fruit setting rate of $5.7 \%$. In combination 2,9 fruits were obtained with a fruit setting rate of $8.4 \%$. In combination 3,5 fruits were obtained with a fruit setting rate of 5.1\%. Through embryo rescue, 126 plants were rescued from combination 1 , 100 plants from combination 2 and 179 plants from combination 3, with a total of 405 plants. However, in the process of transplanting, only 70 plants survived for high temperature and drought, including 25 in combination 1 , 14 in combination 2 and 31 in combination 3 (Table 1).
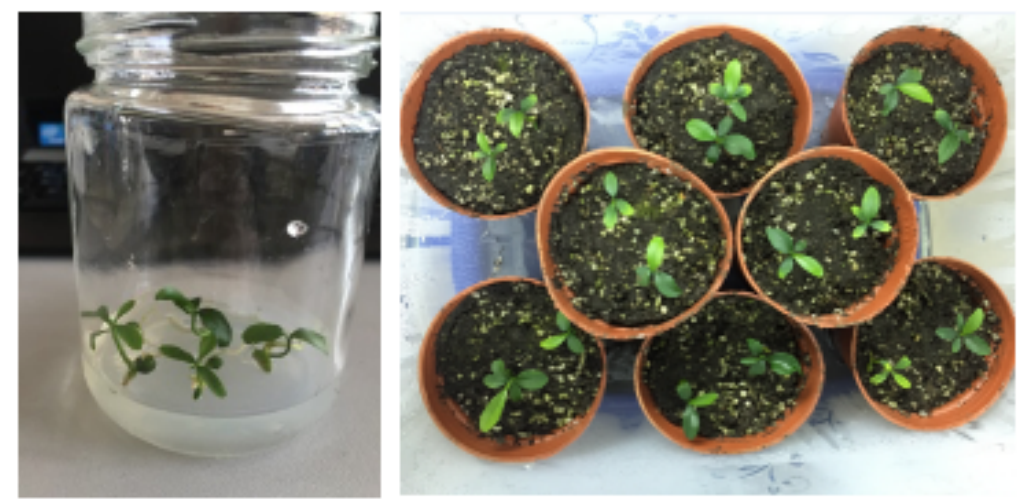

Figure 1 Embryo rescue, plant recovery and transplantation

Note: A: Fruits obtained from hybridization after pollination; B: Seedling in medium; C: The seedling transplantation after acclimatization

Table 1 The seedlings number after Embryo rescue

\begin{tabular}{llllllll}
\hline Crosses & Flower number & Fruit number & Rate of fruit set (\%) & Seed number & $\begin{array}{l}\text { Embryo } \\
\text { number }\end{array}$ & $\begin{array}{c}\text { rescue } \\
\text { Plantet } \\
\text { number }\end{array}$ & $\begin{array}{l}\text { Greenhouse } \\
\text { Number }\end{array}$ \\
\hline Tret1 & 140 & 8 & 5.7 & 72 & 158 & 126 & 25 \\
Tret2 & 107 & 9 & 8.4 & 85 & 179 & 100 & 14 \\
Tret3 & 98 & 5 & 5.1 & 55 & 225 & 179 & 31 \\
\hline
\end{tabular}

\subsection{Flow cytometry detection}

After transplanting, the plant grew to about $20 \mathrm{~cm}$, and the third leaf from top to bottom was taken for flow cytometry detection (Figure 2). The results showed that 3 triploid plants and 1 tetraploid plant were detected by flow cytometry, with triploid rate of $12 \%$ and tetraploid rate of $4 \%$ in combination 1 . In combination 2,3 triploid plants were detected, with triploid rate of $21.42 \%$. In combination 3, 6 triploid plants were detected, with triploid rate of $19.35 \%$. Among the 70 plants, 13 were triploid and tetraploid, and the polyploid rate was $18.57 \%$ (Table 2).

\subsection{Scanning electron microscope of leaf stomata}

The stomata of hybrid tetraploid, triploid and diploid plants were examined by $100 \mathrm{X}$ and $200 \mathrm{X}$ electron microscopy (Figure 3). The results showed that there was no significant difference in stomatal diameter between $4 \mathrm{X}$ hybrids and $3 \mathrm{X}$, and $3 \mathrm{X}$ and $2 \mathrm{X}$ of $\mathrm{n} \times \mathrm{p}$ in combination 1 , but there was significant difference between $4 \mathrm{X}$ and $2 \mathrm{X}$. There was a significant difference between $3 \mathrm{X}$ hybridization and $2 \mathrm{X}$ in combination $2 \mathrm{~K} \times \mathrm{P}$. There was no significant difference between $3 \mathrm{X}$ hybridization and $2 \mathrm{X}$ in combination $3 \mathrm{~B} \times \mathrm{P}$. In terms of the stoma length, there were significant differences between $4 \mathrm{X}$ and $2 \mathrm{X}$ and $3 \mathrm{X}$, but no significant differences between $2 \mathrm{X}$ and $3 \mathrm{X}$ in 
combination $1 \mathrm{n} \times \mathrm{p}$. There was no significant difference between $2 \mathrm{X}$ hybridization and $3 \mathrm{X}$ in combination $2 \mathrm{~K} \times \mathrm{P}$. There was no significant difference between $2 \mathrm{X}$ hybridization and $3 \mathrm{X}$ in combination $3 \mathrm{~B} \times \mathrm{P}$. In terms of stomatal density, there were significant differences between $4 \mathrm{X}$ hybridization and $3 \mathrm{X}$ and $2 \mathrm{X}$, while there were no significant differences between $3 \mathrm{X}$ and $2 \mathrm{X}$ in combination $1 \mathrm{n} \times \mathrm{p}$. There were significant differences between $3 \mathrm{X}$ hybridization and $2 \mathrm{X}$ in combination $2 \mathrm{~K} \times \mathrm{P}$. There were significant differences between $3 \mathrm{X}$ hybridization and $2 \mathrm{X}$ in combination $3 \mathrm{~B} \times \mathrm{P}($ Table 3$)$.

Pearson correlation coefficients showed that the different ploidy was positively correlated with the stoma length, and the ploidy level was 0.348 . Different ploidy was negatively correlated with stomatal density, and the ploidy level was -0.795 (Table 4). In terms of the stomatal characteristics of different ploidy plants, Yi and Rong (2006) studied the leaf genetic variation of sexual progeny of citrus somatic hybridization, and the results showed that the stomatal density of diploid and triploid populations was significantly different, but there was crossover phenomenon in individual plants. The results of this study are consistent with those of previous studies. Tetraploid, triploid and diploid have significant negative correlation in stomatal density, but crossover phenomenon also exists in individual plants.
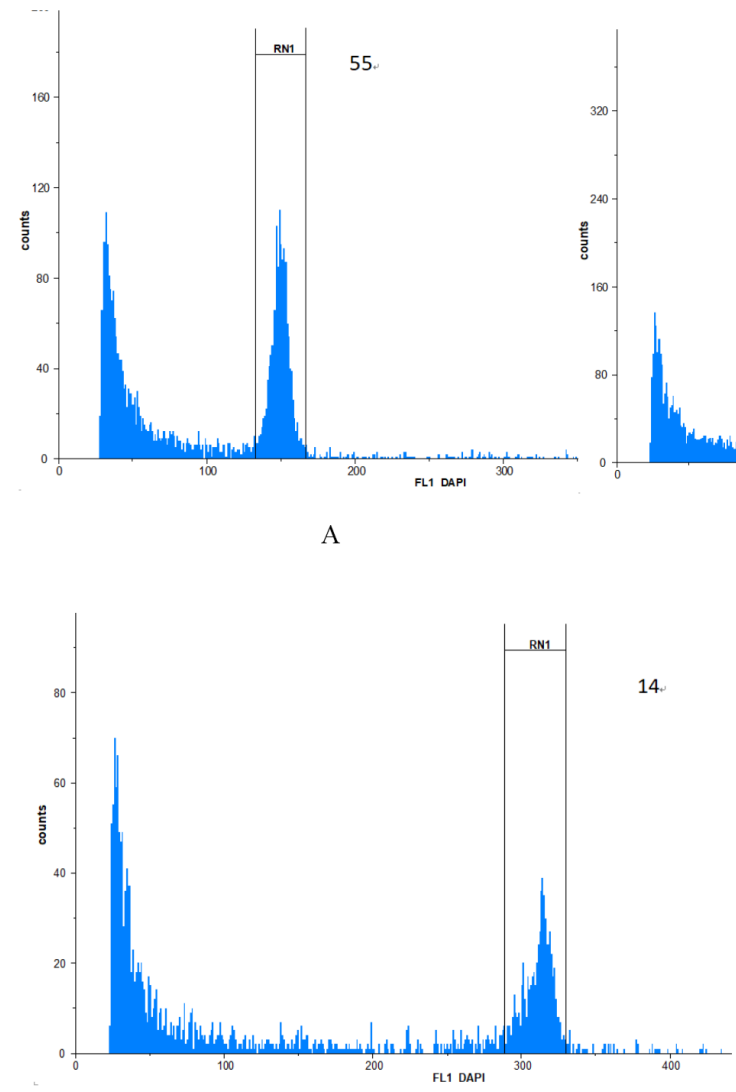

$\mathrm{D}$

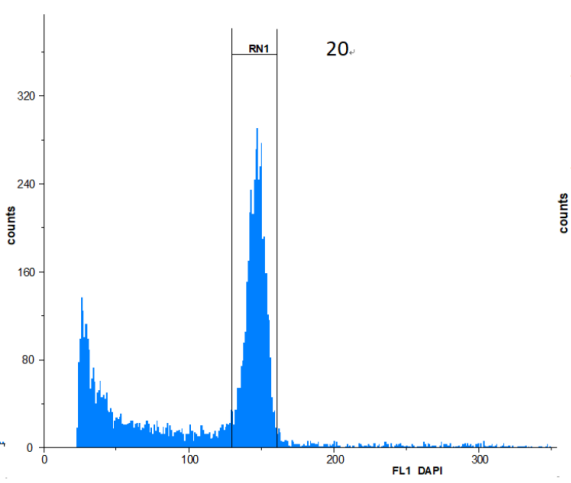

B

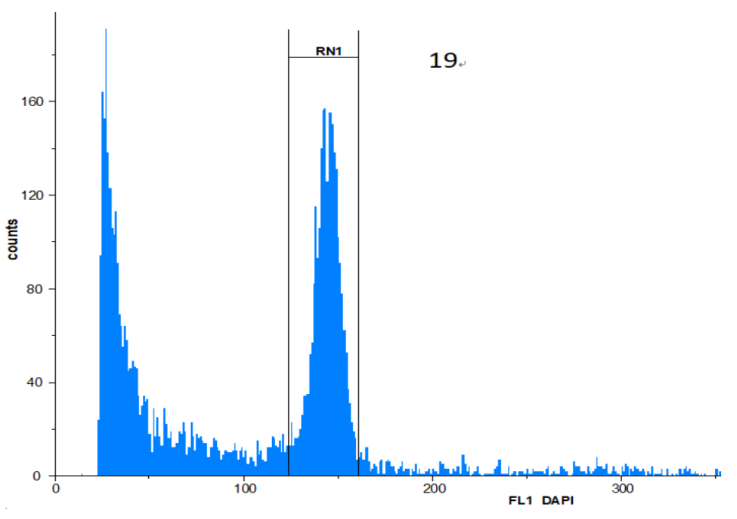

E

Figure 2 Ploidy analysis of Embryo rescue plants by Partec flow cytometry

Note: A: Ponkan CK; B: Tret 1 Triploid plant; C: Tret 2 Triploid plant; D: Tret 3 Triploid plant; E: Tret 1 Tetraploid plant

Table 2 Ploidy analysis of embryo rescue plants by flow cytometry

\begin{tabular}{lllll}
\hline Crosses & Greenhouse number & Diploid number & Triploid number & Tetraploid number \\
\hline Tret1 & 25 & 21 & 3 & 1 \\
Tret2 & 14 & 11 & 3 & - \\
Tret3 & 31 & 25 & 6 & - \\
\hline
\end{tabular}


Molecular Plant Breeding 2020, Vol.11, No.28, 1-8

http://genbreedpublisher.com/index.php/mpb

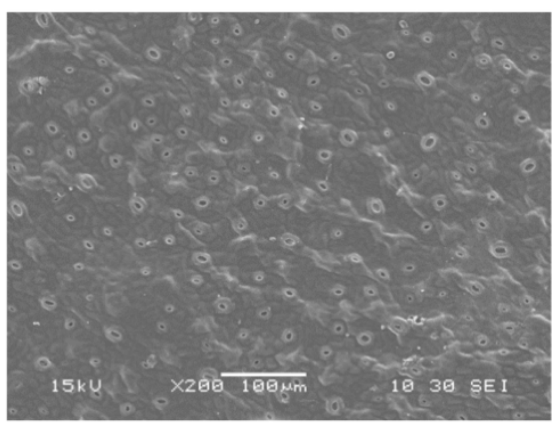

A

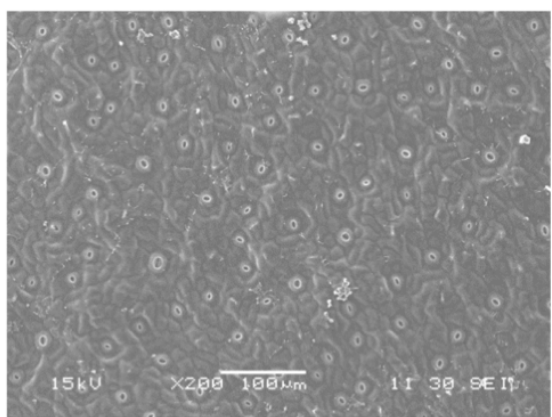

$\mathrm{C}$

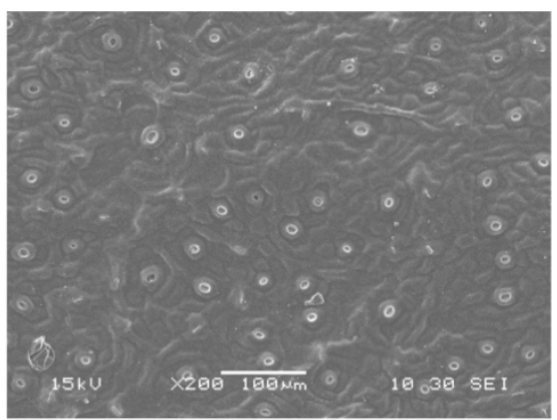

$\mathrm{E}$

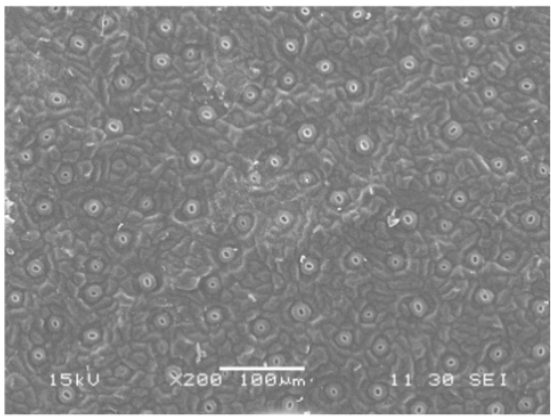

B

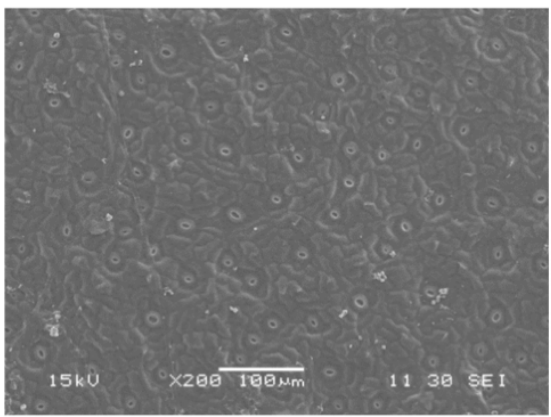

D

Figure 3 Comparison of stoma characteristics among different ploidy seedlings from different combinations (200X) Note: A: Diploid seedlings; B: TRET1 Triploid plants; C: Tret 2 Triploid plants; D: Tret 3 Triploid plants; E: Tret1 Tertraploid plants

Table 3 The embryo rescue plant by stoma electron microscopy

\begin{tabular}{lllll}
\hline Combinations & Ploidy level & Stoma diameter $(\mu \mathrm{m})$ & Stoma length $(\mu \mathrm{m})$ & Stoma density $($ per $/ \mathrm{mm})$ \\
\hline Female & $2 \mathrm{X}$ & $5.76 \pm 1.34 \mathrm{~b}$ & $8.82 \pm 1.08 \mathrm{ab}$ & $405.12 \pm 28.91 \mathrm{a}$ \\
Tret1 & $2 \mathrm{X}$ & $3.71 \pm 1.40 \mathrm{~cd}$ & $7.09 \pm 1.37 \mathrm{~cd}$ & $388.56 \pm 27.59 \mathrm{bcd}$ \\
Tret2 & $2 \mathrm{X}$ & $7.75 \pm 1.22 \mathrm{a}$ & $7.43 \pm 1.17 \mathrm{bcd}$ & $375.40 \pm 42.37 \mathrm{abc}$ \\
Tret3 & $2 \mathrm{X}$ & $3.26 \pm 0.77 \mathrm{~d}$ & $6.38 \pm 1.36 \mathrm{~d}$ & $384.89 \pm 63.80 \mathrm{ab}$ \\
Tret1 & $3 \mathrm{X}$ & $5.41 \pm 2.14 \mathrm{bc}$ & $6.99 \pm 1.04 \mathrm{~cd}$ & $326.86 \pm 32.35 \mathrm{~cd}$ \\
Tret2 & $3 \mathrm{X}$ & $4.83 \pm 1.65 \mathrm{bcd}$ & $8.49 \pm 1.41 \mathrm{abc}$ & $289.41 \pm 40.61 \mathrm{~d}$ \\
Tret3 & $3 \mathrm{X}$ & $4.84 \pm 1.17 \mathrm{bcd}$ & $6.85 \pm 1.04 \mathrm{~d}$ & $285.20 \pm 41.65 \mathrm{~d}$ \\
Tret1 & $4 \mathrm{X}$ & $5.60 \pm 0.54 \mathrm{~b}$ & $9.43 \pm 0.65 \mathrm{a}$ & $185.43 \pm 35.40 \mathrm{e}$ \\
\hline
\end{tabular}

Note: Values within column represent means of five replicates SE; Significance of difference was analyzed via Duncan analysis $(p=0.05)$, same capital letter within column means insignificance 
Molecular Plant Breeding 2020, Vol.11, No.28, 1-8

$\mathrm{http}: / /$ genbreedpublisher.com/index.php/mpb

Table 4 Pearson correlation coefficients between ploidy and stoma characteristic

\begin{tabular}{lllll}
\hline & Stoma diameter & Stoma length & Stoma density & Ploidy level \\
\hline Stoma diameter & - & $0.521^{* *}$ & -0.11 & 0.039 \\
Stoma length & $0.521^{* *}$ & - & $-0.356^{*}$ & $0.348^{*}$ \\
Stoma density & -0.011 & $-0.356^{*}$ & - & $-0.795^{* *}$ \\
Ploidy level & 0.039 & $0.348^{*}$ & $-0.795^{* *}$ & - \\
\hline
\end{tabular}

Note: ${ }^{*}: p<0.05 ; * *: p<0.01$

\subsection{SSR detection}

Five pairs of primers were selected from 36 pairs of primers by polyacrylamide gel electrophoresis (Table 5), and then the parents and their offspring were identified by capillary electrophoresis with these five pairs of primers. The results showed that primer 1 and primer 4 could amplify the differences among Ponkan, Clementine, Nanfeng Mandari and Bingtang orange, while primer 2, 3 and 5 could only amplify the differences between Ponkan and Clementine, but there was no significant difference between Nanfeng Mandari and Bingtang orange. When primer 1 was applied to the parents and offspring by capillary electrophoresis, site deletion was found in offspring 22 and 25. Primer 4 had the highest identification efficiency. The locus size of 64 offspring was the same as that of their female parents. They were all autopolyploid. From left to right, from top to bottom are Ponkan, Clementine, Nanfeng Mandarin; Bingtang Orange, No.1 plant (triploid hybrid offspring of combination 1) and No.25 plant (tetraploid hybrid offspring of combination 1). Primer 4 can well distinguish the differences between the four parents and their offspring (Figure 4).

Table 5 SSR primer information

\begin{tabular}{lll}
\hline Primer name & Primer sequence & Primer sequence \\
\hline 1 & AAAGGGAAAGCCCTAATCTCA & CTTCCTCTTGCGGAGTGTTC \\
2 & GGACCCCTTCAAAGTTTGTTT & AAACGAAAGGACCCAAGTCA \\
3 & CTTACAACACAACTGCTGCAAAGC & TTATAAGTGGGGCCCATCAAAACT \\
4 & AATGATGAGGGTAAAGATG & ACCCATCACAAAACAGA \\
5 & TCTCAATCTCAAATGCACCC & GCACTAGGCTGTGATAAAGGG \\
\hline
\end{tabular}

\section{Discussion}

In this experiment, the regenerated plantlets were obtained by the rescue technology of hybrid embryo between Ponkan and diploid. The results showed that the probability of polyploidy was $18.57 \%$ by flow cytometry and leaf stomatal scanning electron microscopy. This result is much higher than that of $3.12 \%$ triploid plants probability obtained from immature ovules of local early mature fruits reported by Wang and $\mathrm{Li}$ (2007) and $1.559 \%$ tetraploid plants probability obtained from mature Ponkan seeds reported by Hong et al. (2005). It may be that the embryo rescue technology used in this study is different from the previous embryo culture technology. Embryo rescue technology focuses on the immature stage of fruit, through the stripping of immature embryos for in vitro culture and regeneration, rather than stripping undeveloped seeds for in vitro culture after fruit ripening, so as to greatly improve the survival probability of sexual and asexual embryos in polyembryonic citrus seeds, and improve the probability of polyploid acquisition.

The results of this study are consistent with the polyploid rate obtained by Xie et al. (2014) in the new triploid germplasm created by crossing 8 polyembryonic diploid cultivars and allotetraploid, which is about $18 \%$. This result has aroused the research interest of researchers. For a long time, the polyploid produced by citrus hybridization is usually obtained by hybridization of diploid and tetraploid. Although polyploid can be obtained by diploid and diploid hybridization, it is rarely reported. In this paper, the hybrid system of Ponkan and diploid was optimized. The results of flow cytometry and stomatal scanning electron microscope showed that the probability of polyploid produced by the hybrid of Polyembryonic diploid and diploid was about $18 \%$. The results showed that, firstly, Ponkan can effectively create new polyploid germplasm through diploid and diploid hybridization, and the occurrence probability of polyploid is similar to that of Polyembryonic diploid and 
tetraploid hybridization, which not only saves the breeding time, but also shortens the breeding cycles. Secondly, Ponkan can create triploid and tetraploid plants by diploid and diploid hybridization. Hong et al. (2005) and Wang and Li (2007) only obtained tetraploid and triploid plants by embryo rescue of mature seeds, but no report on triploid and tetraploid plants obtained by diploid and diploid hybridization. The results showed that 12 triploid and 1 tetraploid plant were regenerated from the cross between Ponkan and diploid. Thirdly, triploid and tetraploid plants were obtained from the cross between Ponkan and diploid, and the rate of triploid plants was higher than that of tetraploid plants. The above results may be related to the experimental hybrid combination treatment and the characteristics of polyembryonic diploid. It has been reported that the origin of triploids may be due to fertilization of the $2 \mathrm{n}$ oogamete, which may then cease to develop after fertilization (Wang and Li, 2007). Tetraploids may be formed from the doubling of nucellar embryos under natural conditions (Aleza et al., 2011). It is also possible that the $2 \mathrm{n}$ megagamete formed by the female parent stopped dividing after fertilization with the normal diploid pollen to form allotetraploid. The next step is to further verify the formation of triploid and tetraploid, and to further study the formation probability of polyploid. At the same time, triploid and tetraploid resources will be obtained, which will have a certain value for the next step of Ponkan variety improvement.
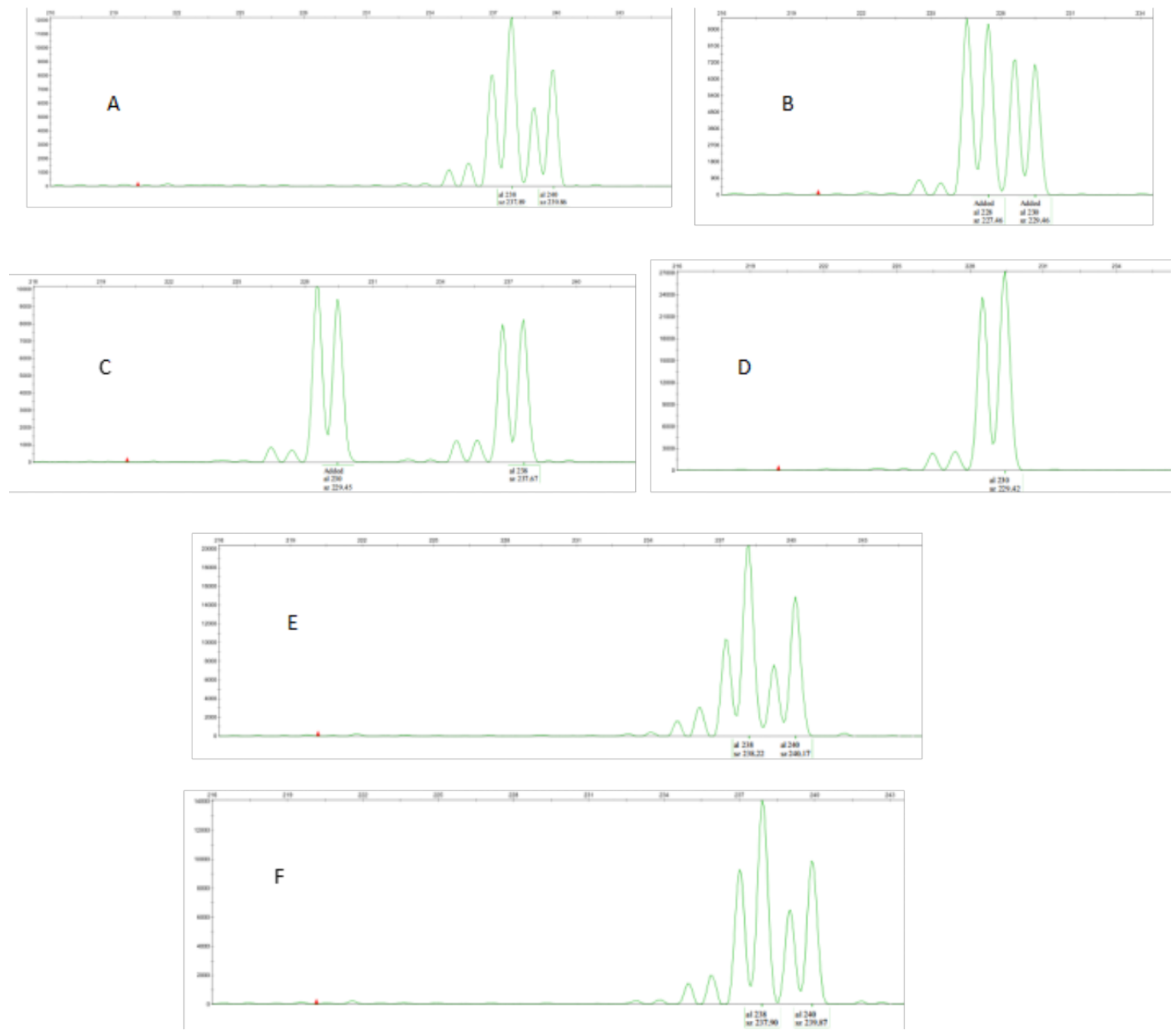

Figure 4 The capillary electrophoretogram of parent materials by primer 4

Note: A: Ponkan; B: Clementine; C: Nanfeng Mandarin; D: Bingtang Orange; E: No.1 plant; F: No.25 plant 
SSR is mainly used to identify whether the offspring is homozygous or heterozygous. Peng et al. (2020) used SSR to identify the tetraploid of Citrange by flow cytometry. The results showed that the amplified bands were completely consistent with the diploid, indicating that the tetraploid obtained were autotetraploid. $\mathrm{n}$ this experiment, 5 pairs of specific primers were screened by polyacrylamide gel electrophoresis. 4 primers screened by capillary electrophoresis can distinguish the specificity between parents and can be used as specific primers for molecular markers of citrus. Five pairs of polymorphic SSR primers were screened to identify the plants of triploid and tetraploid hybrids. The results showed that the regenerated plants were autotriploid and autotetraploid. These naturally discovered polyploid resources are of great value for seedless breeding and related basic research of citrus.

\section{Materials and Methods}

\subsection{Experimental materials}

In April 2017, the pollen of Nanfeng Mandarin (N, diploid), Clementine (K, diploid) and Bingtang orange (B, diploid) were collected in Gaoqiao base of Hunan Horticultural Research Institute as male parents, and crossed with Ponkan 8306 (P, diploid) cultivated in Jishou, as female parents. The unopened flower buds were collected and brought back to the laboratory. Anthers were stripped on filter paper with tweezers and dried at $25^{\circ} \mathrm{C}$. When the pollen was released, the pollen could be collected for the next hybridization experiment.

Three hybrid combinations were designed: combination 1 (Tret1): Nanfeng Mandarin $\times$ Ponkan. Combination 2 (Tret2): Clementine $\times$ Ponkan. Combination 3 (Tret3): Bingtang orange $\times$ Ponkan.

After pollination, the young fruits were picked and stored at $4^{\circ} \mathrm{C}$ in the refrigerator for the next experiment.

\subsection{Embryo rescue}

Refer to the method of Zhang et al. (2013) and modify it appropriately. Under aseptic conditions, the fruits were disinfected with $75 \%$ alcohol, and the seeds were removed from the fruits on the ultra-clean bench. The seed embryos were separated and inserted into the germination medium (MT medium). After 1 month, it was transferred into rooting medium $\left(\mathrm{MT}+\mathrm{GA}_{3} 1.0 \mathrm{mg} / \mathrm{L}\right)$. The culture conditions were as follows: temperature $(25 \pm$ $1)^{\circ} \mathrm{C}$, humidity $(70 \%)$, light $(16 \mathrm{~h})$ per day. After $3 \sim 5 \mathrm{~d}$ of seedling refining, the embryo rescue plants were transferred into the seedling refining device (Zhang et al., 2018). When the plant grew to about $10 \mathrm{~cm}$, the seedlings were transferred and changed into pots. The following experiment was carried out when the height of the seedlings was more than $20 \mathrm{~cm}$.

\subsection{Flow cytometry ploidy detection}

Refer to the method of Xie et al. (2014) and modify it appropriately. With female parent (diploid Ponkan) as contrast, take the first three fully expanded leaf from embryo rescue plants. Cut about $0.5 \mathrm{~cm}^{2}$ of mesophyll tissue with a blade and placed it in a clean plastic petri dish. Added $400 \mu \mathrm{L}$ Lysis Buffer (Paetec HR-A), choped it with a blade, pour it into a $30 \mu \mathrm{m}$ microporous membrane filter tube, and then added $300 \mu \mathrm{L}$ Paetec HR-B solution. After staining, the samples were immediately loaded by flow cytometry (Partec, Cyflow Space, Germany), and the DNA content distribution curve was automatically generated by FloMax software.

\subsection{Scanning electron microscope of leaf stomata}

Refer to the method of Chen et al. (2016) and modify it appropriately. On the same day, the leaves of the third to fourth leaves from top to bottom were collected and dried indoors. The middle part of the leaf was cut into $1 \mathrm{~cm} \times$ $1 \mathrm{~cm}$ small pieces and fixed with FAA fixative solution. The epidermal cells on the back were torn off and put on the sample table. After gold spraying by vacuum plating method, JSW-6380LV (Japan Electron Co., Ltd.) scanning electron microscope was used to observe and take photos with 200X, 500X, 1000X and 2000X. The stomatal size was measured by CAD software, and the average value of 20 observed values was taken. Refer to Yi and Rong (2006) to describe each index of leaf stomata. SPSS21 statistical software was used for statistical analysis. 


\subsection{SSR molecular markers}

According to the SSR primer sequences designed by Zhang et al. (2013) and Xiang et al. (2009), 36 pairs of primers were designed from Sangon Biotech (Shanghai) Co., Ltd. The PCR reaction was $20 \mu \mathrm{L}$ amplification system, including $1 \times \mathrm{PCR}$ buffer, $2.0 \mathrm{mmol} / \mathrm{L} \mathrm{MgCl}_{2}, 0.25 \mu \mathrm{mol} / \mathrm{L}$ bidirectional primers, $0.15 \mathrm{mmol} / \mathrm{L}$ dNTPs, 1.0 U Taq DNA polymerase. The total DNA template was $50 \mathrm{ng}$. Amplification was performed by Applied Biosystems multi-temperature PCR instrument, and the conditions were as follows: predenaturation at $94^{\circ} \mathrm{C}$ for $3 \mathrm{~min}$. Denaturation at $94^{\circ} \mathrm{C}$ for $1 \mathrm{~min}, \mathrm{Tm}$ renaturation for $1 \mathrm{~min}$, elongation at $72^{\circ} \mathrm{C}$ for $1 \mathrm{~min}$, and 35 cycles. Elongation at $72^{\circ} \mathrm{C}$ for $10 \mathrm{~min}$. PCR products were stored in refrigerator at $4^{\circ} \mathrm{C}$. Finally, the amplified products were screened by $8 \%$ polyacrylamide gel electrophoresis.

Further by capillary electrophoresis detection screening best primers. The specific method is as follows: using the parental DNA and offspring DNA as templates, using the SSR primers previously screened, adding $0.5 \mu \mathrm{L}$ amplification products to $10 \mu \mathrm{L}$ mix (HiDi:500 Internal standard=130:1). Capillary electrophoresis was performed on ABI3730 sequencer, and the obtained results were analyzed by Gene Mapper 4.1.

\section{Authors' contributions}

LSN designed and carried out the study, drafted and revised the manuscript. LSN and ZW performed the statistical analysis. CP, HJ, and LFF participated in the design of the study and performed the statistical analysis. LSN and LXX conceived of the project, directed the design of the study, data analysis, draft and revision. All authors read and approved the final manuscript.

\section{Acknowledgments}

This study was supported by the Fund for Hunan Agricultural Youth Innovation (2017QN13) and Modern agricultural Industrial Technology System of Hunan Province (2019-2021).

\section{Reference}

Aleza P., Froelicher Y., Schwarz S., Agusti M., Hernández M., Juárez J., Luro F., Morillon R., Navarro L., and Ollitrault P., 2011, Tetraploidization events by chromosome doubling of nucellar cells are frequent in apomictic citrus and are dependent on genotype and environment, Ann. Bot-London, 108 (1): $37-50$

Chen L.G., and Hu Y.Q., 1981, Study on obtaining triploid from diploid Citrus, Yuanyi Xuebao (Acta Horticulturae Sinica), 8(2): 11

Chen P., Liu J.B., Luo S.N., Bo F.W., Yuan P., Zhang W., and Li X.X., 2016, A study on pollen morphology of pomelo germplasms in hunan province, Yuanyi Xuebao (Acta Horticulturae Sinica), 43(7): 1357-1366

Deng X.X., Guo W.W., and Sun X.H., 1996, Advances in breeding and selection of seedless types of citrus in China, Yuanyi Xuebao (Acta Horticulturae Sinica), 23(3): $235-240$

Deng X.X., 2005, Advances in worldwide citrus breeding, Yuanyi Xuebao (Acta Horticulturae Sinica), 32(6): 1140-1146

Guo W.W., Yi H.L., and Deng X.X., 1997, In vitro culture and citrus triploid breeding, Zhongguo Nanfang Guoshu (South China Fruits), 26(6): 11-13

Hong L., Liu Y.Z., and Deng X.X., 2005, Obtaining of ponkan (citrus reticulate blanco) tetraploid by culturing embryos of mature seeds, Yuanyi Xuebao (Acta Horticulturae Sinica), 32(4): 688-690

Peng Y., Li X.Y., and Xiao X., 2020, Excavation and SSR identification of autotetraploids in citrus polyembryonic rootstock Citrange', Fenzi Zhiwu Yuzhong (Molecular Plant Breeding), 18(4): 1211-1215

Wang Z.C., and Li Z.A., 2007, Obtaining triploid citrus plant via culturing undeveloped ovules of ripen fruits, Henan Daxue Xuebao (Journal of Henan University (Natural Science)), 37(2): 174-176

Xiang S.Q., He J., He B., Wang W.X., Li X.L., Guo Q.G., He Q., and Liang G.L., 2009, Genetic diversity of polyploids of Shatianyou pomelo by SSR markers, Guoshu Xuebao (Journal of Fruit Science), 26(3): 382-385

Xie K.D., Wang X.P., Wang H.Q., Liang W.J., Xie Z.Z., Guo D.Y., Yi H.L., Deng X.X., Grosser J.W., and Guo W.W., 2014, High efficient and extensive production of triploid citurs plants by crossing polyembryonic diploids with tetraploids, Yuanyi Xuebao (Acta Horticulturae Sinica), 41(4): 613-620

YI H.L., and Deng X.X., 1998, Study on culturing citrus triploid plantlets with somatic hybrid as male parent, Yichuan (Hereditas), 20: 47-49

Yi H.L., and Rong R., 2006, Study on the heredity and variation of leaf morphological character of sexual progenies of the citrus somatic hybrid, Guoshu Xuebao (Journal of Fruit Science), 23(2): 169-172

Zhang W., 2013, The hybrids obtainment of citron C05 resistant to canker disease and the identification by molecular, Hunan Nongye Daxue (Changsha: Hunan Agricultural University), pp.44

Zhang W., Hu W., Zhang X.Y., Zhou M., Jiang Q.Q., Deng Z.N., and Li D.Z., 2013, Acquisition of hybrids of pummelo*citron by embryo rescue and their identification by SRAP molecular markers, Guoshu Xuebao (Journal of Fruit Science), 30(3): 386-389

Zhang W., Luo S.N., Chen P., Han J., Li F. F., Li X.X., and Li D.Z., 2018, The invention relates to a citrus embryo rescue seedling specialized refining seedling box, Patent no: ZL 201821975420.1

Zhou K.L., and Ye M.M., ed., 2010, Chinese fruit tree*citrus roll, Beijing: China forestry press,Beijing China, pp.2 